\title{
Hospitalisation und Absonderung in Pestzeiten - die Schweiz im Vergleich zu Oberitalien
}

\author{
Von Vera Waldis
}

Die oberitalienischen Städte Venedig und Mailand waren zur Zeit der großen Pestepidemien Europas führend in deren Bekämpfung. Unter anderem, weil die fundierteren Rechtsgrundlagen eine gut organisierte Verwaltung und ein straffes Polizeiwesen mit sich brachten. Gerade das auch bei uns in der beginnenden Neuzeit erstarkende Polizeiwesen war für die Pestbekämpfung wichtig.

Seit dem «Schwarzen Tod» (1347-1352) erweiterten Venedig und Mailand mit jeder neuen Epidemie die staatlichen Prophylaxemaßnahmen. Die Isolation der Kranken, die Quarantäne für krankheitsverdächtige Personen, die Reinigung (Purga) verdächtiger $W$ aren, die absolute Passagesperre (Bando) für Kaufleute aus infizierten Orten, alle diese Einrichtungen sind im wesentlichen in Oberitalien während des 15. Jahrhunderts entwickelt worden ${ }^{1}$. Dabei ist zu beachten, daß diese Maßnahmen großenteils gegen eine allfällige Ansteckung gerichtet waren. Die italienischen Stadtstaaten setzten sich damit über die theologische Lehre, die Pest sei eine unausweichliche Strafe Gottes, hinweg und ließen sich auch nicht durch die in der Medizin vorherrschende Theorie beirren, nach welcher die Pest ihre Ursache in einer Luftverderbnis (Miasmalehre) hatte. Erst 1546 wurde von einem Teil der Mediziner die verfeinerte Kontagionslehre des Girolamo Fracastoro $^{2}$ (1478-1553) übernommen.

Venedig, das sich nur gegen eine Einschleppung der Seuche auf dem Seeweg wehren mußte, hatte es einfacher als der Binnenstaat Mailand, der sämtliche zuführenden Straßen und Wege zu kontrollieren hatte. Und da die Sperren, die Mailand errichtete, nicht ausreichend waren, versuchte es im 16. Jahrhundert auch auf die Pestbekämpfung der Nachbarstaaten Einfluß zu nehmen. So wurde mit starkem handelspolitischem Druck die Eidgenossenschaft verpflichtet, auf eigenem Gebiet dieselben Abwehrmaßnahmen zu ergreifen. Mailand behielt sich dabei die Oberaufsicht vor. Die Eidgenossen unterzeichneten den entsprechenden Vertrag $1585^{3}$, ohne sich des eigentlichen Grundes - der Kontagiosität der Pest - bewußt zu sein. Auch der Stadtschreiber Luzerns, Renward Cysat (1545-1614), der sich seit 1580 mit 
der staatlichen Pestbekämpfung beschäftigte ${ }^{4}$, stieß erst durch die Pestabhandlung des Ensisheimer Stadtarztes Georges Garnier $(1550-1614)^{5}$ auf die Kontagionslehre. Er resümiert die 1610 erschienene Abhandlung: «Er [Garnier] wie auch andere Medici concludirend stark, daß der Lufft diser Zyt unverändert vnd für nit vergriffen, sondern ein accidentalische pestis sye, vnd dz Contagium allein durch das Commertium vnd Mangel scharpffen Uffsehens yngebracht werde.» ${ }^{6}$ Wir ersehen aus dieser Stelle, daß Garnier wie viele seiner Zeitgenossen die Miasmatheorie neben der Kontagionslehre bestehen ließ: Einmal konnte die Ursache für eine Pestepidemie in der Luftvergiftung liegen, ein andermal, wie «diser Zyt» (1610), war das Contagium der Grund.

Je nach Gültigkeit der Miasma- oder Kontagionslehre bekommt auch die Hospitalisation der Pestkranken eine andere Bedeutung. Solange die Miasmalehre vorherrscht, hat die Hospitalisation allein den Zweck, Kranken, die aus irgendeinem Grund nicht von ihren Angehörigen gepflegt werden können, eine Wartung zu garantieren. Die hiebei entstehenden Kosten werden vornehmlich durch karitative Spenden gedeckt. Nimmt man aber an, daß die Pest ansteckend, also vom Kranken auf den Gesunden übertragbar sei, wird es für die Allgemeinheit wichtig, daß jene von den Gesunden isoliert werden. Die Hospitalisation erlangt damit politisches Interesse, die Kosten werden so immer mehr dem Fiskus aufgelastet. Neben den Kranken mußten auch die anderen potentiellen Pestgift-Überträger isoliert werden. Als solche wurden die an der Pest Genesenen und die Personen, die mit Pestkranken in Kontakt kamen, betrachtet.

Nur zur Pflege hospitalisiert wurden 1564 die Pestkranken im damals vorderösterreichischen Rheinfelden. Das Ratsprotokoll hält fest, daß die adelige Frau von Schönau ${ }^{7}$ nach feudalistisch-mittelalterlichem Prinzip der ansonst recht autonomen Stadtverwaltung den Auftrag gab, für die Kranken zu sorgen. Selbst wollte sie dies mit einem Almosen von 100 Pfund unterstützen:

«Vff disen thag hatt Herr Vmgeldter meinen herren von wegen der altenn frawenn von Schenauw angezeigt das $B i$ für sguot anseche, die weil die sterbende leuff verrhandenn das meine herren, guote Ordnung vff die Armann habenn, vnd ein Siechenpflegerr jnn der Armenn leit New huis ${ }^{8}$, oder jnn Spital ordnenn söllen. Deruff Bi $100 £$ erlegt.» ${ }^{9}$

Der Rat beschloß, die Kranken nicht im Siechenhaus, sondern im Spital unterzubringen ${ }^{10}$. Es handelte sich bei den Aufgenommenen meist um 
Bedienstete der Stadtbürger. Obwohl das Almosen der Frau von Schönau eigentlich für einige Zeit hätte ausreichen sollen, wurde auch der Besitz der Erkrankten zur Kostendeckung herangezogen:

«Vff disenn thag ist erkhennt Die weil sich bey disenn Sterbendenn leiffen begebenn, das dienst Knecht, vnd megt krankh werdenn, jnn Spittal gelegt alda $\beta j$ erhaldtenn, doch soll von einer jeden person was die hatt vnnd vermag vberandtwurt werdenn, So denn die selbige person mitt thod abgeen würde, also dann so soll, den Erbenn so schein bringen, das jhenig zuhandenn gesteldt darus aber zuvorderst grebdt, warthung vnd derglichen endtricht werden, Diß ist am heuit datto gegenn denenenn vonn Baßelln, die weil ein Magt von dennenn byrthig verrschidenn, gevbt, vnd was es verlassen zugesteldt.. ${ }^{11}$

In einer öffentlichen Spitalordnung wurde noch beigefügt, daß jeder Bürger bei seinem Eid und jede Witwe «bey jrenn pflichten» genaue Auskunft über das Vermögen und eventuelle Guthaben ihrer Bedienten Auskunft geben müsse ${ }^{12}$.

Das erste permanente Pestlazarett in Venedig, das 1423 auf der Insel S. Maria die Nazareth - daher der Ausdruck Lazarett - errichtet worden war, nahm ebenfalls nur Pestkranke auf ${ }^{13}$, dies im Gegensatz zur bekanntlich ersten Quarantänestation von Ragusa, die 1377 für pestverdächtige Ankömmlinge zu deren Reinigung (ad purgandum) erstellt worden war ${ }^{14}$. Das «Lazzaretto Nuovo», das Venedig 1468 zusätzlich bauen ließ, war ursprünglich für die Rekonvaleszenten aus dem «Lazzaretto Vecchio» geplant, von denen man annahm, daß sie noch bis zu vierzig Tagen das ansteckende Gift mit sich herum tragen könnten ${ }^{15}$. Doch diente das neue Lazarett seit seiner Fertigstellung (1471) auch zur Absonderung von Gesunden, die unter dem Verdacht standen, daß sie in Kontakt mit dem Pestgift gekommen seien. Falls jemand aber hier erkrankte, wurde er sofort ins Lazzaretto Vecchio übergeführt ${ }^{16}$. Beide Lazarette leisteten in den großen Epidemien von 1575-1577 und 1630 gute Dienste. Im 18. Jahrhundert wurden die Bauten arg vernachlässigt. Als John Howard (1726-1790), der Philanthrop, der sich für Neuerungen im Gefängniswesen, aber auch für Verbesserungen in den Pestlazaretten einsetzte, 1785 nach Venedig kam, mußte er ebenfalls eine Quarantäne durchmachen, da er absichtlich auf einem Schiff mit schlechtem Gesundheitsschein ankam. Als bei ihm Kopfschmerzen auftraten, wurde er vom neuen Lazarett ins alte überführt, ohne daß aber eine schlimmere Erkrankung sich erzeigt hätte. Nach seiner Beschreibung waren die beiden 
Lazarette damals in furchtbarem Zustand; die Räumlichkeiten erregten nach seiner Schilderung wahren Ekel und schienen ihm menschenunwürdig. Hier wie dort ließ er seine Räume aus eigenen Mitteln übertünchen und instandsetzen ${ }^{17}$. Die venezianischen Lazarette wurden immer zu einem großen Teil von der öffentlichen Hand finanziert. Zu Beginn unterstanden sie dem über große Mittel verfügenden «Magistrato al Sal». ${ }^{18}$

An sich wäre es für die Verminderung der Ansteckungsgefahr ideal gewesen, wenn ein allgemeiner Hospitalisationszwang für Kranke und Verdächtige hätte statuiert werden können. Aus Platzgründen war dies aber nie durchführbar. Immerhin, 1576 wurden in Venedig verschiedene Projekte zur vollständigen Trennung von Kranken und Gesunden entworfen: Nach einem dieser Projekte sollte auf der Terraferma eine riesige Zeltstadt für die Gesunden aufgestellt werden. Venedig wäre so zu einem einzigen großen Lazarett geworden ${ }^{19}$.

Ähnliche Verhältnisse wie in Venedig finden wir für die Pest von 1576 (Peste di S. Carlo) auch in Mailand. Allerdings ist die Dokumentation über die Entwicklung der Lazarettbauten Mailands viel spärlicher, da 1502 die Kanzlei des 1447 schon bestehenden «Ufficio della Sanità» vollständig niederbrannte ${ }^{20}$. Gesichert ist, daß 1451 das erste Lazarett Mailands in Cusago entstand ${ }^{21} .1576$ gab es mehrere Pesthospitäler und bei S. Gregorio eine Quarantänestation. Hier wurden am Schluß der Epidemie die Überlebenden der übrigen Lazarette bis zur völligen Genesung zurückbehalten ${ }^{22}$. Die ersten Quarantänen wurden 1476 verfügt ${ }^{23}$.

Auf eidgenössischem Gebiet bildeten die Städte des Waadtlands eine Ausnahme. Sie hatten noch unter dem Hause Savoyen vorbildliche Maßnahmen erlassen. Nachdem es von Bern erobert wurde (1536), baute es sie noch weiter aus, obwohl die Berner Direktiven dies nicht verordneten. Lausanne verlangte von den kranken, infizierten und verdächtigen Einwohnern der Stadt (malades, infects, suspicionnés), daß sie ihre Wohnung räumten und die Stadt verließen. Hauseigentümern blieb es freigestellt, ob sie die sechswöchige Quarantäne zu Hause oder außerhalb der Stadt durchmachen wollten ${ }^{24}$.

Die katholischen eidgenössischen Stände begannen nach den italienischen Vorbildern in der ersten Hälfte des 17. Jahrhunderts ebenfalls Pestspitäler zu bauen und ähnliche Isolationsmaßnahmen für Kranke und Verdächtige zu treffen. Sie waren den protestantischen Orten voraus, da sie von Mailand stärker beeinflußt waren und mehr unter Druck gesetzt wurden. Luzern baute 1596 sein erstes permanentes Pestspital ${ }^{25}$, und 1611 wurden 
verschiedene Häuser außerhalb der Stadt als Quarantänestationen in Gebrauch genommen ${ }^{26}$. In Zürich finden wir lange Zeit nur Ansätze solcher Maßnahmen. 1564 und auch später wurden die Pestkranken im ehemaligen Klostergebäude im Selnau untergebracht ${ }^{27}$, hingegen kam es nie zur Einrichtung eines Sperrhauses für Gesunde, die mit Kranken in Kontakt kamen. Hingegen überlegte sich 1668 der Rat - wie 1468 in Venedig -, ob nicht Baulichkeiten für Rekonvaleszenten bereitgestellt werden sollten ${ }^{28}$.

An diesem Nachhinken der Protestanten war die Prädestinationslehre nicht ganz unschuldig. Denn ob die Gelehrten Kontagionisten oder Anhänger der Miasma-Lehre waren, über eines war nicht zu streiten: Prinzipiell lag die Ursache der Pest letztendlich immer bei Gott: Er bediente sich der «natürlichen Mittel» - Miasma oder Kontagium -, um die sündige Menschheit zu strafen. Die Anhänger der Prädestinationslehre sahen sich deshalb nicht veranlaßt, etwas gegen die Pest zu unternehmen: Wollte Gott sie damit schlagen, dann traf er sie, wollte er sie verschonen, blieben sie gesund. Martin Luther (1483-1546) wandte sich in seinem «Bericht: Ob man für dem Sterben vnd zur Pestilenszeit fliehen müge?» (1533) mit gewohnter Heftigkeit gegen eine solche Einstellung. Seiner Meinung nach sind diese

«... allzu vermessen vnd keck/also/ daß sie Gott versuchen/ vnd lassen alles anstehen/damit sie dem Sterben oder Pestilentz wehren solten/ verachten Ertzney zu nehmen/ vnd meiden nicht Stette vnd Person/so die Pestilentz gehabt vnd aufkommen sind/ sondern zechen vnd spielen mit ihnen/ wöllen damit ihre freidigkeit beweisen/ vnnd sagen/ Es sey Gottes Straffe/ wölle er sie behüten/ so wird ers wohl thun/ ohn alle Ertzney vnd vnsern fleis/ Solchs heist nicht Gott trawen/ sondern Gott versuchen $/ \ldots$ Wer derselben [Arznei] nicht braucht/... der verwarloset seinen Leib selbs vnd sehe zu/ daß er nicht sein selbs Mörder erfunden werde für Gott. ... Zu dem ist das noch grewlicher/ daß ein solcher/so seinen Leib also verwarloset vnd der Pestilentz nit hilfft wehren/ so viel er kan/ möchte damit auch viel andere beschmeissen vnd vergifften/ ... würde also schuldig seines Nehesten Todes/ vnd vielmal für Gott ein Mörder.» ${ }^{29}$

Für viele Reformierte galt es auch als Verletzung der christlichen Pflichten, wenn sie ihre Kranken einfach ins Hospital verfrachteten. Bezeugt wird dies 1565 vom württembergischen Reformator Johannes Brenz (1499-1570): 
«Sie halten auch/ es sey wider die Liebe des Nächsten/ daß eine weltliche Obrigkeit Verordnung thue/ damit die Krancken an der Pestilentz von Gesunden abgesondert/ vnd die andern/ so aus sterbenden Orthen kommen nicht eingelassen werden/ ... ${ }^{30}$

Solche Überlegungen mochten auch in Basel lange ihren Vorrang gehabt haben: Erst 1668, als die Epidemie sich schon lange in der Stadt ausgebreitet hatte, stellte der Sanitätsrat seine «Bedenckhen wegen beschließung der Heüßeren» an. Um möglichst bald die Wiedereröffnung der Handelswege für die Basler Kaufleute zu erlangen, schlug er vor:

«daß nemblich ein Jedes hauß, sobalden Jemandt, wehr der auch währe, darin ahn dießer Leidigen Seüche erkranckht, von Obrigkheits wegen beschlosßen, die darin sich befündtliche gesundte, vndt Kranckhe ahn gewüsße von E: Gn: darzue bestimmende Ortth, Alß daß bereits habende Lazaret, vnd etwan ein, vndt ander Kloster; so hierzue accomodiert werden müste, ohne Mittel verschafft vndt so wohl die,: welche Kranckh, oder gesundt dahinkommen, alß auch die, welche widerumb aldorthen geneßen, biß nach außgestandener vierzig Tägiger Quarantaine von der geneßung, darin abgesünderet, ...».

Wohl aus finanziellen Gründen stimmte der Rat von Basel diesem Vorschlag nicht zu, da nur ein Teil der Mittel aus dem Spitalalmosen genommen werden sollte, der Rest aber bei den «vermöglichen widerumben zuerholen» gewesen wäre ${ }^{31}$.

Somit waren Kostenfragen, Handelsinteresse, medizinische und theologische Überlegungen, die Organisation des Staatswesens mitbestimmend bei den verschiedenen Fragen über die Hospitalisation von Pestkranken. Die Lazarette mußten drei Funktionen gerecht werden: der Pflege der Pestkranken, ihrer Isolation und der Absonderung gesunder potentieller Pestüberträger. In Italien wurde dies verwirklicht, während hierzulande erst später diese Einsicht gewonnen wurde. 


\section{Anmerkungen}

${ }^{1}$ Zur staatlichen Pestprophylaxe in Mailand und Venedig:

- Giussani, Achille, L’Archivio del Magistrato della Sanità in Milano. Annuario del Archivio di Stato in Milano (1915).

- Palmer, Richard J., L'azione della Repubblica di Venezia nel controllo della peste. Lo sviluppo della politica governativa.

- Zitelli, Andreina, L'azione della Repubblica di Venezia nel controllo della peste. Lo sviluppo di alcune norme di igiene pubblica. Venezia e la peste 1348/1797, ed. Comune di Venezia, Assessorato alla Cultura e Belle Arti, Venezia 1979, S.103-110 und 111-112.

${ }^{2}$ Fracastorus, Hieronymus, De Contagione. Opera omnia, Venedig 1555.

Fracastoro nennt drei mögliche Ansteckungsarten: Direkte Berührung (105v); Aquirieren der Pestsamen, nachdem diese über bestimmte Zeit in einem sie konservierenden Gegenstand (Betten, Kleider, Holz u. a.) erhalten worden waren (106r-107r); auf Distanz (105v).

${ }^{3}$ Staatsarchiv Basel, San. Q 1,1. CAPITULATIONE Co $i$ Signori Suizzeri, intorno alle occorenze della Sanità. Dat. in Belinzona il Lunedì alli 12. Agosto 1585 [gedruckte Version].

${ }^{4}$ Cysat veranlaßte 1580, daß die Luzerner Behörden Pestverordnungen publizierten. Vgl. Brunner-Wildisen, Bianca, Medizinisches aus den Schriften des Renward Cysat 1545-1614. Zürcher Medizingeschichtliche Abhandlungen Nr.138, Zürich 1980, S. 13.

${ }^{5}$ Garnerus, Georgius, Epitome de peste, Pruntrut 1610. Garnier stammte aus Pruntrut, wurde Leibarzt des Herzogs Maximilian von Österreich, der ihm die Verwaltung seiner Güter in Deutschland anvertraute. 1613 wurde ihm der erbliche Reichsadel verliehen. (HistorischBiographisches Lexikon der Schweiz, Bd.3, Neuenburg 1926, S.399.)

${ }^{6}$ Zit. n. Schnyder, Franz, Pest und Pestverordnungen im alten Luzern, Diss. med. Basel 1932, S. 55 .

${ }^{7}$ Katharina von Schönau, †12.3.1585. Geb. von Rechberg von Hohenrechberg, Witwe des Hans Ludwig von Schönau, Rat und Landvogt des Erzherzogs Ferdinand von Österreich in Ortenau. Sie machte verschiedene karitative Stiftungen. Vgl. Schib, Karl, Geschichte der Stadt Rheinfelden, Rheinfelden 1961, Tf. 15, S. 160 und S.290.

${ }^{8}$ Sondersiechenhaus. Vgl. Burkart, Sebastian, Geschichte der Stadt Rheinfelden, Aarau 1909, S.698, Anm. 3.

${ }^{9}$ Stadtarchiv Rheinfelden (StRh), Ratsprotokoll (RP), 6.7.1564.

${ }^{10} \mathrm{StRh}$ RP, 15.7.1564.

${ }^{11}$ StRh RP, Montag nach Margarethe [20.7.] 1564.

12 StRh RP, 15.9.1564.

${ }^{13}$ Müller, Reinhold C., Dalla reazione alle prevenzione, Venezia e la peste ... op. cit., S.84-85.

${ }^{14}$ Sticker, Georg, Abhandlungen aus der Seuchengeschichte und Seuchenlehre, 2 Bde., Gießen 1908-1912. I,2 Die Pest, S. 295.

15 Venezia e la peste ..., op. cit., Anhang S. 366.

${ }_{16}$ Palmer, op. cit., S. 104.

17 Howard, John, Nachrichten von den vorzüglichsten Krankenhäusern und Pesthäusern in Europa, Leipzig, bey Georg Joachim Goeschen, 1791, S. 22-24.

18 Palmer, op. cit., S. 104-105.

${ }_{19}$ Preto, Paolo, Peste e Società a Venezia nel 1576. Studi e testi veneziani 7. Vicenza 1978, S. 158-159. 
${ }^{20}$ Giussani, Achille, L'Archivio del Magistrato della Sanità in Milano. Annuario del R. Archivio di Stato in Milano (1915), S. 139 und 144.

21 ibid. S. 141, Anm. 1.

${ }^{22}$ La Cava, A. Francesco, La peste di S. Carlo. Contribuiti della Scuola di Storia della Medicina dell'Università di Milano (1944), S. 162 und 191.

${ }^{23}$ Giussani, op. cit., S. 142.

${ }^{24}$ Olivier, Eugène, Médecine et santé dans le pays de Vaud des origines à la fin du XVII siècle. Bibliothèque historique vaudoise, 29/30, 2 Bde., Lausanne [1962]. Bd.2., S. 607-611.

${ }^{25}$ Schnyder, op. cit., S. 53-54.

26 ibid. S. 70.

${ }^{27}$ Wehrli, Gustav Adolf, Die Krankenanstalten und die öffentlich angestellten Ärzte und Wundärzte im alten Zürich. Mitteilungen der Antiquarischen Ges. in Zürich, Bd. 31/3 (1934), S. 33-35.

${ }^{28}$ Ruesch, Hanspeter, Der Beitrag der Zürcher Ärzte zur obrigkeitlichen Pestbekämpfung (1519-1721). Zur Publikation vorbereitete Maschinenschrift, S. 137-138.

${ }^{29}$ Zit. n. der Ausgabe «Erfurdt/Jn verlegung Johan Birckners/ ...» 1626, S. 16-17.

${ }^{30}$ Zit. n. der Ausgabe: Osiander, Andreas, \& Brentius, Johannes, «Zwey Theologische CONSILIA Oder Bedencken/ 1. Andreae Osiandri, ... 2. Joh. Brentii, wie man sich in Sterbens $=$ läufften $/$ zur Zeit der Pestilentz/Christlich verhalten soll?», Jena 1680.

${ }^{31}$ Staatsarchiv des Kantons Aargau, Reg. Nr.6376; «Fricktal; Gesundheitswesen: Anstekkende Krankheiten (Contagion) 1593-1794»: «Bedenckhen ... verleßen den 21.9bris i668.» [Zeitgenössische Kopie.]

\section{Summary}

In plague epidemics since the fifteenth century, the north Italian cities of Venice and Milan used to isolate affected persons as well as potential plague carriers. In the States of the Swiss Confederation, in spite of a convention with Milan on plague prevention along the Gotthard route (1585), contagiousness of plague was not recognized before the beginning of the 17th $\mathrm{c}$. Until then, hospitals were used only for nursing plague patients, not for isolating them in order to protect the community. Measures to prevent the importation and spread of plague were taken earlier and more vigorously by the catholic Swiss States than by the protestant ones. In Lucerne a first permanent plague hospital was built in 1596, and quarantine was introduced in 1611.

Dr.med.Vera Waldis

Medizinhistorisches Institut

Rämistraße 71

CH-8006 Zürich 